Recebido em 22.07.2011. Aprovado em 22.10.2012 Este artigo tem coautoria de membro do Corpo Editorial Científico da RAE.

foi avaliado em double blind review, com isenção e independência. Editores Científicos: Carlos Osmar Bertero, Flavio Carvalho de Vasconcelos, Marcelo Pereira Binder, Thomaz Wood Jr

\title{
PRODUÇÃO CIENTÍFICA BRASILEIRA EM GESTÃO DE PESSOAS NO PERIODO 2000-2010
}

BRAZILIAN SCIENTIFIC PRODUCTION IN HUMAN RESOURCE MANAGEMENT IN THE PERIOD 2000-2010

PRODUCCIÓN CIENTÍFICA BRASILEÑA SOBRE GESTIÓN DE PERSONAS EN EL PERÍODO 2000-2010

\section{RESUMO}

$\mathrm{O}$ artigo analisa quatro aspectos dos textos em gestão de pessoas: sua contribuição, referencial teórico, procedimentos metodológicos e discussão. A análise abrange os artigos publicados nos periódicos nacionais de maior impacto, entre 2000 e 2010 . O objetivo é esclarecer suas fragilidades diante das políticas edi- toriais preconizadas por top journals, em português e inglês, e apontar direções para evolução. Para evoluir qualitativamente nesta década, a discussão sugere implicações à prática acadêmica e uma agenda de pesquisas visando alcançar impacto, rigor e relevância da produção em Administração no Brasil.

PALAVRAS-CHAVE Ensino e pesquisa em administração, produção científica, impacto, rigor acadêmico, gestão de pessoas.

André Ofenhejm Mascarenhas amascarenhas@fei.edu.br

Professor do Departamento de Administração, Centro Universitário da FEI - São Paulo - SP, Brasil

Allan Claudius Queiroz Barbosa allan@ufmg.br

Professor do Departamento de Ciências Administrativas, Universidade Federal de Minas Gerais - Belo Horizonte - MG, Brasil

Abstract This article examines four key factors in texts on human resource management: their contribution, theoretical framework, methodological procedures and discussion. The analysis covers articles published in Brazilian top periodicals from 2000 to 2010. The purpose of this inquiry is to clarity their weaknesses in face of editorial policies advocated by top journals in Portuguese and English, and to suggest directions to evolution. To make qualitative progress during this decade, the discussion suggests implications on academic practice and a research agenda aimed at achieving impact, rigor and relevance of scientific production in business administration in Brazil.

keywords Reserch and education in business administration, scientific production, impact, academic rigor, human resource management.

Resumen El artículo analiza cuatro aspectos de los textos sobre gestión de personas: su contribución, referencial teórico, procedimientos metodológicos y discusión. El análisis abarca los artículos publicados en los periódicos nacionales de mayor impacto, entre 2000 y 2010. El objetivo es aclarar sus fragilidades ante de las politicas editoriales preconizadas por top journals, en portugués e inglés, y señalar direcciones para evolución. Para evolucionar cualitativamente en esta década, la discusión sugiere implicaciones en la práctica académica y una agenda de investigaciones con el objetivo de lograr impacto, rigor y relevancia en la producción sobre administración en Brasil.

Palabras clave Enseñanza e investigación en administración, producción cientifica, impacto, rigor academico, gestión de personas. 


\section{INTRODUÇÃO}

Consolidou-se, nos diferentes campos da Administração, o debate sobre qualidade da produção científica, que sugere consensos razoáveis sobre suas limitações. O aumento quantitativo não estaria relacionado à maior qualidade da produção, que seria pouco relevante e rigorosa, frágil, voltada à academia e pouco preocupada com aplicabilidade (BERTERO, CALDAS, WOOD JR, 1999, 2005; BERTERO, 2007; TONELLI e outros, 2003). O debate sobre a evolução da pesquisa em Administração no Brasil, suas fragilidades e as oportunidades para consolidar o campo nos próximos 10 anos ganha relevo, considerando as possibilidades que se abrem no campo qualitativo.

Para contribuir com o debate, propõe-se aprofundar a discussão sobre o impacto, o rigor e a relevância da produção (MASCARENHAS, ZAMBALDI, MORAES, 2011; MATTOS, 2012). Nessa perspectiva, são analisados quatro aspectos dos artigos em Gestão de Pessoas entre 2000 e 2010: sua contribuição, referencial teórico, procedimentos metodológicos e discussão. A análise abrange os textos publicados nos periódicos nacionais de maior impacto. O objetivo é esclarecer as fragilidades dos textos diante das políticas editoriais preconizadas por top journals, em português e inglês, e, assim, apontar direções para evolução.

A análise baseia-se em editoriais (GEPHART, 2004; SUDDABY, 2006; VASCONCELOS, 2009) e inspira-se em uma linha analítica que investiga como os autores constroem a consistência dos textos publicados (LOCKE e GOLDEN-BIDDLE, 1997; BARTUNEK e RYNES, 2010). Recupera-se a abordagem pioneira de Bertero, Caldas e Wood Jr. (1999), que dava voz a periódicos e avaliadores notáveis ao propor critérios objetivos para mensurar qualidade. A discussão sugere implicações à prática acadêmica e uma agenda de pesquisas visando ao impacto, ao rigor e à relevância da produção em Administração no Brasil.

\section{METODOLOGIA DE ANÁLISE DA PRODUÇÃO EM GESTÃO DE PESSOAS (2000-2010)}

A evolução qualitativa da produção em Administração depende de aprofundarmos o debate sobre seu impacto. Segundo Judge e outros (2007), o impacto de um texto acadêmico depende do prestígio do periódico, o que sugere a necessidade de o pesquisador compreender os caminhos para a publicação em top journals e, em especial, dominar o ciclo da produção científica: geração de ideias, construção da teoria e escrita clara.

O recorte empírico justifica-se pela pertinência do debate. A produção acadêmica em Gestão de Pessoas no Brasil tem sido marcada por desafios inerentes a um campo em transformação. Área historicamente associada ao fortalecimento da Administração, viu, na última década, um movimento pendular, ora permitindo ampliação de seu escopo pela ênfase interdisciplinar, abertura e fragmentação temática, ora restringindo-se, com o deslocamento de conteúdos para outras áreas ou mesmo a criação de espaços específicos derivados, tal como o debate comportamental com lógica própria. Enquanto área sujeita às dificuldades comuns às demais áreas da Administração, o campo vem sendo objeto de reflexão. Esses trabalhos revelam limitações da produção, direcionando sua atenção para a análise propositiva, contributiva para um salto de qualidade (TONELLI e outros, 2003; CALDAS e TINOCO, 2004; BARBOSA, 2009; MEDEIROS e OLIVEIRA, 2009).

Também, as particularidades do campo refletem os fundamentos do modelo analítico, que enfatiza contribuições teóricas e práticas. A análise do "mapa da partilha" da antiga área de recursos humanos, no âmbito da Associação Nacional de Programa de Pós-Graduação em Administração (ANPAD), após seu desmembramento em 2001, indica que Gestão de Pessoas concentrou temas com viés mais evidente para a aplicação prática, diante das áreas correlatas de relações de trabalho e estudos organizacionais (TONELLI e outros, 2004). Além disso, a pertinência das proposições reveladas pela análise acaba, também, por sinalizar à orientação afeita ao mainstream científico ou às próprias condições objetivas para o trabalho acadêmico de ponta. É nesse sentido que a análise assume maior abrangência, dialogando com tendências em pesquisa e publicação de outros campos da Administração no Brasil.

O modelo analítico, apresentado a seguir, fundamentou-se em editoriais e documentos recentes (reviews, cartas editoriais, políticas, entrevistas) de periódicos nacionais e anglófonos prestigiosos em management e Gestão de Pessoas (buman resource management). Nos últimos anos, vários editoriais discutem as expectativas de rigor e relevância dos periódicos (GEPHART, 2004; SUDDABY, 2006; VASCON- 
CELOS, 2009). Na arena anglófona, foram reunidos editoriais do Academy of Management Journal, Academy of Management Review, Human Resource Management Journal, Journal of Organizational Behavior e Journal of Management Studies, além de pareceres e cartas editoriais do Journal of Applied Behavioral Science, Organization Science e International Journal of Cross-Cultural Management. Entre os periódicos em português, foram selecionados editoriais e debates, constantes das referências, além de pareceres da RAE-Revista de Administração de Empresas (RAE) e da revista Organizações \& Sociedade.

\section{Contribuição do artigo}

O primeiro aspecto analisado foi se e como os autores posicionam seu artigo diante da literatura, explicitando, nas introduções, sua contribuição. Formular um problema de pesquisa consiste em situá-lo, contextualizá-lo e diagnosticá-lo (VAN DE VEN, 2007). Ao fazê-lo, a contribuição de um trabalho seria o que este traz de novo e único, perante o problema, seja o desenvolvimento teórico e/ou metodológico ou a melhor compreensão dos fenômenos (LOCKE e GOLDEN-BIDDLE, 1997).

A relevância do texto científico surgiria do reconhecimento social da pertinência do problema e do valor de sua contribuição. Assim, seria necessário o reconhecimento subjetivo do potencial da contribuição do artigo, no âmbito do processo editorial, para a publicação por periódicos de prestígio (BEYER, CHANOVE, FOX, 1995; RYNES, 2002). Dada a crescente competição por publicação, Ashkanasy (2009) sugere que não haveria sentido em submeter um artigo que não explicite, logo de início, o que traz de novo e por que tal contribuição merece atenção (justificativa da contribuição).

Para justificar a contribuição, seria necessário construir a aderência dos objetivos aos diálogos da literatura atual, posicionando-os explicitamente em termos de sua pertinência e importância. Ao investigar contribuições em estudos organizacionais, Locke e Golden-Biddle (1997) mostram que os autores recuperam um mosaico de referências para caracterizar e, então, problematizar uma literatura.

\section{Referencial teórico}

O segundo aspecto analisado foi a consistência do referencial teórico. Construir o referencial teórico im- plica integrar e justificar conhecimentos explanatórios ao problema de pesquisa, tornando-se uma espécie de lente pela qual se analisa a problemática (VAN DE VEN, 2007). Diversos editoriais recentes tratam da qualidade do referencial teórico. Ashkanasy (2010) sugere que estudos da tradição positivista/quantitativa apresentem uma sólida base teórica, e não somente um apanhado de estudos empíricos anteriores e hipóteses. Gephart (2004) discute a importância de os estudos qualitativos estarem bem fundamentados, teórica e metodologicamente, na abordagem de pesquisa adotada. Para o editor, estudos qualitativos e quantitativos devem explicitar suas perguntas de pesquisa, definir seus termos e revelar sua perspectiva teórica, criando consistência entre teoria, pergunta e metodologia.

A construção cuidadosa e criativa do referencial teórico sugere rigor do trabalho científico. Ao enfatizarem sua natureza explanatória, Sutton e Staw (1995) alegam que referências, dados, listas de variáveis e construtos, hipóteses e diagramas não poderiam ser considerados teoria. Referências fazem menção à teoria, dados revelam o que aconteceu, variáveis e construtos não revelam por si o porquê de suas relações, hipóteses presumem o que será observado, enquanto diagramas não substituem uma explicação textual do porquê das relações alegadas. Whetten (2003) descreve os blocos constitutivos de uma teoria, incluindo o que ou quais variáveis, construtos ou conceitos devem ser considerados, como esses elementos conceituais estão relacionados, introduzindo causalidade, e o porquê ou a lógica que justifica as relações propostas e unifica a teoria.

\section{Procedimentos metodológicos}

O terceiro aspecto analisado foi o conteúdo das seções de metodologia. O rigor de um texto científico implica construção conceitual e teórica cuidadosa, além da observância a ditames metodológicos (MATTOS, 2008; VASCONCELOS, 2009). O reconhecimento do rigor de um trabalho acadêmico depende da apreciação da qualidade e pertinência do conjunto de decisões sobre métodos, abrangendo a consistência das escolhas teórico-metodológicas. A estrutura geral do trabalho, articulada pela tríade problema - teoria - metodologia, revela a abordagem de pesquisa adotada, enquanto a seção de metodologia revela as decisões tomadas e executadas, bem como as integra e justifica no contexto geral da pesquisa.

Procedimentos metodológicos bem-definidos reve- 
lam transparência no planejamento e na execução da pesquisa. Pratt (2009) e Gephart (2004) afirmam que muitos enfatizam o irrelevante, omitindo questões de fato pertinentes, como: (1) explicar por que a pesquisa é necessária, (2) definir a abordagem genérica à metodologia e por que é adequada, (3) explicar se está sendo elaborada teoria nova ou existente, (4) explicar quais são e o porquê de ter escolhido contexto e unidades de análise, (5) explicar como os dados foram coletados e que práticas transformaram observações em resultados e conclusões. O detalhamento seria importante para que não se tenha que "comprar" as conclusões.

\section{Discussão}

O quarto aspecto analisado foi como os textos fazem sua contribuição nas seções de discussão. A contribuição teórica deve ser significativa (CLARK, FLOYD, WRIGHT, 2006) ou forte (PRATT, 2009), e são os insights à teoria pela falsificação de entendimentos convencionais, elaboração indutiva de teoria, primeiro teste empírico de teoria, meta-análises com implicações teóricas e a replicação construtiva que clarificam a abrangência de teoria (EDITORIAL TEAM, 2007). Além disso, uma contribuição teórica sólida implica discutir as oportunidades abertas. Estudos sem contribuição teórica ou essencialmente descritivos, e o uso de casos para ilustrar ou aplicar uma teoria existente, não justificam a publicação em top journals (SUTTON e STAW, 1995).

A análise identifica também como os artigos fazem sua contribuição prática, emergindo daí a relevância da pesquisa aplicada (VAN DE VEN, 2007). Reconhecemos o debate complexo em torno dessa posição (JARZABKOWSKI, MOHRMAN, SCHERER, 2010; STARKEY e MADAN, 2001; GREY, 2001; BARTUNEK, 2007; WEICK, 1996). Porém, a análise segue editores para quem a lacuna entre pesquisa e prática é excessivamente grande (RYNES, 2007; RYNES, BARTUNEK, DAFT, 2001). Para Clark, Floyd e Wright (2006), os autores devem refletir sobre as implicações de suas ideias no cotidiano da Administração.

\section{RECORTE EMPÍRICO E PROCEDIMENTOS DE ANÁLISE}

A construção da base de artigos partiu da decisão de privilegiar os periódicos, excluindo a produção em congressos. Assim, o estudo não pretendeu abranger com exatidão o mapa da produção da área no período, mas, sim, o estado da arte da produção na década passada. A decisão considera, ainda, que, mesmo que os pesquisadores eventualmente direcionem seus textos a outros periódicos, em inglês ou de outras áreas, haveria pertinência na análise da produção nacional nos periódicos de Administração com maior impacto. Além disso, excluir a produção em congressos viabilizou a análise qualitativa proposta.

Os periódicos selecionados foram aqueles considerados de maior impacto entre os nacionais, medido pelo índice $\mathrm{H}$, conforme estudo da $R A E$ (2012). Esse critério fez a escolha recair sobre RAE, RAUSP e RAC. O recorte é consistente com o estudo desenvolvido por Machado-da-Silva e outros (2008) sobre o impacto de periódicos brasileiros de Administração entre 2005 e 2007, e com trabalhos anteriores (TONELLI e outros, 2003; MEDEIROS e OLIVEIRA, 2009). O recorte abrange 32 artigos, em português, em Gestão de Pessoas, resumidos no Quadro 1.

Para o enquadramento dos artigos, considerou-se, em primeiro lugar, a aproximação dos textos às definições de subtemas da Anpad. Considerou-se, também, a ênfase em gestão, verificada por meio da incidência da palavra no título, palavras-chave, resumo e, em poucos casos, texto completo. Embora Tonelli e outros (2004) revelem sobreposições temáticas que dificultam a classificação, várias rodadas de discussão excluíram da amostra os artigos que traziam ambiguidades pela aproximação com as áreas de relações do trabalho ou estudos organizacionais. Cada artigo foi submetido a leitura, análise textual e de conteúdo, conforme detalhamento no Quadro 2.

\section{DISCUSSÃO E IMPLICAÇÕES À PRÁTICA ACADÊMICA}

A análise dos quatro aspectos dos artigos em Gestão de Pessoas entre 2000 e 2010 buscou esclarecer fragilidades dos textos diante das políticas editoriais preconizadas por topjournals, e, assim, apontar direções para aperfeiçoamentos nesta década. É nesse sentido que a análise revelaria uma proposta para balizar a produção acadêmica artesanal.

A análise mostra que, nas introduções, predominam os objetivos dos artigos. Menos prevalente é a contextualização e justificação dos objetivos 
pela literatura. Assim, confundem-se objetivos com contribuição. Alguns artigos não fazem referência à literatura relevante atualizada. Mesmo quando há citações, as introduções são imprecisas e não permitem avaliar a pertinência do texto, pois não identificam tendências na literatura. Em vez disso, os objetivos são frequentemente contextualizados por referências genéricas à globalização, dinamismo do mercado e tendências funcionais, empregando retórica dominante em RH ("O ser humano como principal ativo da organização"), reproduzindo o que Wood Jr., Tonelli e Cooke (2011) denominam discurso da neocolonização em RH.

A análise revela a dificuldade de os autores sistematizarem sua contribuição. Nesses artigos, o esforço para justificar ou contextualizar os objetivos não revela o que o estudo agrega. Por exemplo, justificativa usual foi alegar poucos estudos, ou a introdução do tema nos estudos brasileiros, sem refletir adequadamente sobre a pertinência de se trilharem os caminhos propostos. Uma recomendação é que as introduções recuperem e sistematizem os estudos prévios, para clarificar e contextualizar as fronteiras do diálogo, revelando como se pretende fazê-lo avançar. Amadurecimento das ideias seria revelado pelas convergências e divergências na literatura, sobre as quais um estudo se assentaria. Artigos que justificam sua contribuição de maneira mais direta revelam esforços sistemáticos dos autores para aprofundar temas, com cumulatividade das pesquisas.

\section{Quadro 1 - Recorte empírico por periódico}

\begin{tabular}{|c|c|c|c|}
\hline Periódicos & $\begin{array}{c}\mathrm{N} . \\
\text { artigos }\end{array}$ & $\begin{array}{c}\text { Instituição e ano de } \\
\text { publicação dos artigos }\end{array}$ & Temas abordados \\
\hline$R A E$ & 13 & $\begin{array}{l}\text { UNB, 2001; EAESP/FGV, 2001; EAESP/FGV, 2003; } \\
\text { UFRGS, 2003; EAESP/FGV, 2004; UNB, 2004; UNB, } \\
\text { 2005; UNISINOS, 2006; EAESP/FGV, 2006; FDC, } \\
\text { 2008; PUCMG e UFMG, 2006; EAESP/FGV e BSP, } \\
\text { 2008; UFSJ e UFMG, 2010. }\end{array}$ & $\begin{array}{l}\text { Seleção de pessoas, gestão da mudança, avaliação de } \\
\text { treinamento, impacto do treinamento, desenvolvimento de } \\
\text { competências, gestão de competências, políticas e práticas } \\
\text { de RH, gestão do desempenho, identidade social, gestão } \\
\text { estratégica de RH, universidade corporativa, abordagem } \\
\text { institucional, gestão da diversidade. }\end{array}$ \\
\hline$R A C$ & 12 & $\begin{array}{l}\text { USP, 2000; UNB, 2000; USP, 2001; UNB, 2003; } \\
\text { UNB, 2003; FGV-EBAPE, 2004; FGV-EAESP, 2005; } \\
\text { UFMG, FUCAPE e UFES, 2008; UPF/RS e UFRGS, } \\
\text { 2009; UFSJ/MG, 2009; PUC/MG, Insead/França e } \\
\text { Faculdade Pedro Leopoldo/MG, 2010; FGV-EAESP, } \\
2010 .\end{array}$ & $\begin{array}{l}\text { Gestão da remuneração, gestão por competências, impacto } \\
\text { do treinamento, competência, gestão da motivação, carrei- } \\
\text { ras, sistemas de informação em RH, políticas de RH, cultura } \\
\text { corporativa, gestão internacional de pessoas, gestão da } \\
\text { diversidade, gestão estratégica de pessoas, expatriação. }\end{array}$ \\
\hline RAUSP & 7 & $\begin{array}{l}\text { UNB, 2003; UNB, 2003; UNIMONTES, 2003; } \\
\text { UFMG, 2003; EAESP/FGV e USP, 2005; USP, 2006; } \\
\text { UNINOVE e IMES, } 2010 .\end{array}$ & $\begin{array}{l}\text { Impacto de treinamento, avaliação de treinamento, demis- } \\
\text { são, gestão de competências, avaliação e mensuração em } \\
\text { Gestão de Pessoas, contrato psicológico, qualidade de vida } \\
\text { no trabalho. }\end{array}$ \\
\hline
\end{tabular}

\section{Quadro 2 - Detalhamento da análise dos artigos - questões abordadas}

Aspecto

1. Contribuição

2. Referencial teórico

3. Metodologia

4. Discussão

\section{Análise}

0 artigo explicita e justifica sua contribuição? Quais são seus objetivos, sua contribuição e justificativa? 0 texto identifica tendências na literatura? Essa literatura é recuperada? 0 que é citado?

Hipóteses/pergunta de pesquisa emerge de construção teórica consistente? 0 artigo alega referencial teórico, abrangendo o quê, como e por quê (SUTTON e STAW, 1995; WHETTEN, 2003)?

Qual é a estratégia metodológica? Há detalhamentos e justificativas para o objeto de pesquisa, unidades de análise e para os procedimentos metodológicos?

0 artigo alega construção ou reelaboração de teorias segundo as modalidades de Editorial Team (2007)? É possível identificar os blocos constitutivos da teoria (Whetten, 2003)? Há agenda para estudos futuros? Discutem-se limitações do estudo? 0 artigo traduz achados em implicações à prática? 
Quanto ao referencial teórico, poucos artigos alegaram explicitamente recuperar ou integrar abordagens teóricas. Em vez disso, o uso de levantamentos conceituais, tipologias, taxonomias, contextualizações históricas, estudos empíricos anteriores e conteúdos prescritivos como teoria reforça o caráter descritivo dos referenciais teóricos. Essas seções referem-se a uma variedade de perspectivas e definições, sem articulá-las em conhecimentos explicativos com base nos quais se construiria a coerência do levantamento empírico. Nesses artigos, a conexão entre teoria e metodologia é frágil. A pertinência do levantamento empírico não emerge da revisão teórica, pouco capaz de iluminar e justificar a estratégia metodológica. Por outro lado, os artigos que propõem referenciais teóricos com viés explicativo fazem-no predominar diante das reflexões geradas pelo levantamento empírico, o que fragiliza o texto, ao minimizar sua contribuição original.

A análise da seção de metodologia revelou as estratégias de pesquisa mais frequentes: estudos de caso, levantamentos survey submetidos à análise multivariada, estudos de validação de escalas, análise de dados qualitativos e estratégias quali-quanti em estudos exploratórios e descritivos. Principalmente em artigos qualitativos, apreciações conceituais sobre a metodologia prevalecem perante as justificativas e decisões sobre procedimentos, que aparecem com menor detalhamento. Por exemplo, em estudos de caso, apresentam-se fundamentos da estratégia, em vez de justificativa adequada que revele a pertinência do caso e o conecte à problemática, o que enfraquece a estratégia.

No lugar de justificativas que integrem e contextualizem as decisões em métodos, esses artigos alegam procedimentos como se preenchessem um formulário quase padronizado, o que é distinto de explicitar e integrar os desafios enfrentados e as decisões adotadas em seus aspectos estratégicos, táticos e relacionais. Por exemplo, muitos estudos enfatizam procedimentos como a gravação e transcrição das entrevistas, mas não revelam a lógica e pertinência da seleção dos entrevistados nem o percurso da construção das categorias e relações com base no material empírico; ou, ainda, mencionam o uso de documentos como parte de táticas de triangulação, sem revelar quais e como se complementam à construção da análise.

Quanto à discussão, o que caracteriza a produção em Gestão de Pessoas é o raciocínio dedutivo, e a maioria dos trabalhos visava ao desenvolvimento de teorias existentes, principalmente por meio do teste empírico que expande a teoria ou meta-análises com implicações teóricas. Porém, os artigos não se posicionam quanto à sua contribuição, mesmo que, com frequência, isso se revele ao longo do texto. Quando alegada, é comum que a contribuição do artigo coincida com seus objetivos e/ou com os objetivos do levantamento empírico, sugerindo contextualização insuficiente dos objetivos pela literatura ou, ainda, pouca reflexão com base nos dados analisados. Por exemplo, é comum, na seção final, o autor retomar os objetivos para declará-los cumpridos e ensaiar uma reflexão sobre a pertinência do esforço analítico. Essas deficiências dificultam identificar o caráter de novidade das relações estabelecidas e discutir potencialidades.

Sobre a estrutura das contribuições, alguns textos são descritivos, exibindo riqueza de dados, mas pouca análise. Outros assumem linguagem que os aproxima de relatórios de consultoria, com prejuízos ao rigor científico, por não enfatizarem o processo analítico que sustentaria a argumentação. Alguns artigos tinham clara pretensão prescritiva, revelavam consistência no processo analítico, sem, contudo, aprofundar suas implicações práticas ou contextualizá-las e explorar suas potencialidades e limites. Quando a discussão era teórica, os textos não aprofundavam seus desdobramentos, apesar de estes serem a essência da contribuição nesses casos.

Sobre agenda futura ou limitações do estudo, ideias genéricas são mencionadas e não revelam reflexão comprometida com a linha de raciocínio desenvolvida. Por exemplo, é comum citar a "necessidade de mais estudos", o que não convence o leitor sobre o porquê de tais estudos serem necessários e que caminhos seriam potencialmente promissores. Sobre as limitações do estudo, é comum mencionar-se a "impossibilidade de generalização" quando a metodologia não se presta a isso. O Quadro 3 sumariza os elementos problematizados ao longo da análise.

\section{LIMITAÇÕES DO ESTUDO E AGENDA DE PESQUISAS FUTURAS}

Este fórum relança o debate da qualidade da produção científica brasileira em Administração, ao propor a reflexão sobre sua evolução na última década, suas fragilidades e oportunidades para consolidar o campo nos próximos 10 anos. Com base em trabalhos pioneiros da década de 1990, balanços da produção foram 


\section{Quadro 3 - Principais fragilidades dos textos em Gestão de Pessoas}

\begin{tabular}{|l|l|}
\hline \multicolumn{1}{|c|}{ Aspecto } & \multicolumn{1}{c|}{ Fragilidades } \\
\hline Contribuição & $\begin{array}{l}\text { Introduções genéricas e imprecisas. } \\
\text { Prevalência de objetivos, com raras referências à contribuição. } \\
\text { Dificuldade de os autores sistematizarem tendências na literatura. }\end{array}$ \\
\hline \multirow{2}{*}{ Referencial teórico } & $\begin{array}{l}\text { Predomínio de abordagens descritivas e conceituais. } \\
\text { Conexão frágil entre teoria e metodologia. } \\
\text { Referenciais teóricos extensos minimizam contribuição original do texto. }\end{array}$ \\
\hline Metodologia & $\begin{array}{l}\text { Justificativas insuficientes. } \\
\text { Alegação formal de procedimentos. }\end{array}$ \\
\hline Discussão & Pouco detalhamento do percurso metodológico. \\
\hline & Discussão insuficiente. \\
& Pouco comprometimento com agenda futura. \\
Alegações genéricas quanto a limitações dos estudos.
\end{tabular}

publicados em todas as subáreas da Administração, retratando a expansão da academia e consolidando uma série de reflexões que são a base do debate sobre o desejado salto de qualidade. Entre essas reflexões, preferência deveria ser dada a estudos empíricos executados segundo metodologias rigorosas e que revelem aplicabilidade (BERTERO e KEINERT, 1994; BERTERO, VASCONCELOS, BINDER, 2003), permitam teorização (BERTERO, CALDAS, WOOD JR, 1999; TONELLI e outros, 2003) ou a crítica habilitada (DAVEL e ALCADIPANI, 2002). Entretanto, este artigo revela a prevalência de abordagens com baixa pretensão metodológica, sendo parte da produção limitada a estudos de caso exploratórios, o que é condizente com análises anteriores (TONELLI e OUTROS, 2003), e sugere pouca evolução na última década.

Neste ponto, questão central que esta análise recoloca é: por que as fragilidades identificadas persistem? O que fazer para saná-las e avançar? Este debate não é novo, já que vários autores vêm discutindo as competências para a produção acadêmica artesanal (RODRIGUES, 2004; ROESCH, 2003; CARVALHO e VIEIRA, 2003; SERRA, FIATES, FERREIRA, 2008). Contudo, a academia em Administração ainda não se comprometeu com análises sistemáticas de como os pesquisadores levam a cabo os empreendimentos acadêmicos, segundo uma abordagem da academia como prática (ver, por exemplo, FREITAS, 2011; MATTOS, 2012). As práticas de priorizar, definir, caracterizar perguntas e abordagens de pesquisa, contextualizar e traduzir as reflexões acadêmicas não seriam conformadas somente pelas pressões normativas do "publicar ou perecer", mas também por práticas e fatores adjacentes, como as políticas das instituições de ensino e pesquisa, os comprometimentos simultâneos na carreira, a formação dos pesquisadores e sua inserção nas comunidades. A academia como prática como agenda de pesquisa contribuiria para o olhar crítico ao produtivismo, aprofundando a reflexão sobre as estratégias empregadas por pesquisadores e instituições e que fortalecem a academia. Apesar das pressões do "publicar ou perecer", haveria espaço para melhor priorização de projetos de pesquisa, viabilizando a dedicação artesanal aos textos (ALCADIPANI, 2011; MATTOS, 2012).

Para contribuir com o debate da qualidade da produção científica brasileira em Administração, as implicações à prática acadêmica reveladas por esta análise da produção em Gestão de Pessoas podem também orientar a produção em outros campos da Administração que compartilhem entendimentos quanto às condições objetivas para o trabalho acadêmico de ponta. Entre as limitações desta análise, em ciências sociais aplicadas, o critério de relevância abrangeria necessariamente referenciais externos à academia, e a noção de rigor revelada pela proposta estaria, ainda, submetida a outras convenções acordadas nas comunidades científicas, com implicações adicionais para a construção dos artigos. Por exemplo, em etnografia, o rigor estaria associado à capacidade de $\mathrm{o}$ texto convencer o leitor experiente, abrangendo aspectos como autenticidade, plausibilidade e criticidade (GOLDEN-BIDDLE e LOCKE, 1993). Em estudos de múltiplos casos, o rigor diz respeito à capacidade de o estudo permitir a construção de teoria por meio da replicação entre casos (EISENHARDT, 1991). Atenção a essas convenções também fortaleceria a produção brasileira em Administração. 
Este artigo sugere que produzir ciência social aplicada com excelência resulta no impacto da pesquisa. Assim, seria profícuo direcionar atenção ao impacto da pesquisa em Administração: o que é e como se constrói? Aqui se deve ressaltar a limitação da noção de impacto adotada nesta análise. Segundo a concepção de Judge e outros (2007), o impacto revela-se pela referência em forma predominante de citações ao trabalho científico por outro, na esfera estritamente acadêmica, o que obscurece o potencial transformador dos textos em esferas não acadêmicas (VAN DE VEN, 2007). Apesar disso, essa noção estreita reflete o entendimento majoritário sobre mensuração do impacto da produção, gerando críticas (ILGEN, 2007). Como agenda futura, propõe-se aprofundar a discussão sobre como construir impacto, rigor e relevância da produção científica, deslocando atenção dos acadêmicos da diversidade temática, epistemológica e demográfica da produção para os processos de engajamento, construção e disseminação inerentes ao ciclo de produção científica (MASCARENHAS, ZAMBALDI, MORAES, 2011; MATTOS, 2012). Apesar de sua importância, os balanços da produção brasileira não avançaram explicitamente a reflexão sobre como promover diálogos na sociedade e construir agendas relevantes de pesquisa.

Como ciência aplicada, o impacto da produção em Administração depende de se compreender a disposição da sociedade a incorporá-la como matéria-prima para a reflexão e ação transformadora, constatação que expõe a necessidade de agenda de pesquisa específica. Por exemplo, apesar de viabilizar maior impacto da pesquisa, as atividades de contextualizar e traduzir reflexões acadêmicas são esforços da carreira que precisam ser compreendidos e estimulados. Esse desafio já vem sendo enfrentado em outras arenas científicas. Entre os tópicos atuais, discute-se como o conhecimento acadêmico se preza ao uso na prática (NICOLAI e SEIDL, 2010) e em sala de aula (JARZABKOWSKI e WHITTINGTON, 2008), a natureza e a dinâmica das interações entre acadêmicos e praticantes (BEECH, MACINTOSH, MACLEAN, 2010; KNIGHTS e SCARBROUGH, 2010), entre outros. Desafios ao debate incluem a busca de definições adequadas aos conceitos de conhecimento, teoria e relevância, e às suas relações sistemáticas com a ação e a prática, além de dificuldades teóricas e empíricas no desenvolvimento de teorias sobre a geração, transferência e uso do conhecimento (JARZABKOWSKI, MOHRMAN, SCHERER, 2010, p. 1191).
Construir o impacto da produção brasileira em Administração dependeria de se aprofundar o entendimento sobre sua relevância. Mattos (2012) e outros já colocaram a questão: para quem seria a produção relevante? Dois caminhos para reflexão emergiriam. O primeiro seria analisar para quem escrevemos. Segundo Spink e Alves (2011), o dilema de instituições e pesquisadores seria, na prática, privilegiar certas agendas com as quais se engajar. Diante da tendência pela internacionalização, esse dilema se resumiria à escolha entre a produção de relevância às agendas anglófonas, concebida implicitamente como agenda internacional, mas relevante principalmente aos países de língua inglesa, e a produção de relevância local, que refletiria o modelo da universidade comprometida com a sociedade civil, que direciona atenção à problemática local sem descuidar de processos globais subjacentes. Segundo caminho seria analisar o que escrevemos. Aqui valeria a proposição de Rousseau, Manning e Denyer (2008), para quem somente a síntese de um corpo de evidências científicas revelaria implicações consistentes à prática, abrangendo acúmulo, análise e interpretação de um conjunto de textos. Dado o volume atual da produção científica nacional, seria desejável identificar e sistematizar a contribuição dos textos, sintetizar o conhecimento sendo acumulado, refletir sobre seus limites e desdobramentos, e indicar caminhos para desenvolvimento futuro.

\section{NOTA DE AGRADECIMENTO}

Os autores agradecem aos professores Otávio Sanches, Felipe Zambaldi, Flávio Carvalho de Vasconcelos, Patrícia Mendonça, José Mauro da Costa Hernandez, Charles Kirschbaum e Rafael Alcadipani pelas críticas e sugestões. Agradecem também aos professores Reinaldo Mora e Vanessa Cabral pelo apoio na construção da base de dados e análises. E também aos editores e avaliadores da $R A E$, cujo aconselhamento foi inestimável ao aperfeiçoamento do texto.

\section{REFERÊNCIAS}

ALCADIPANI, R. Resistir ao produtivismo: uma ode à perturbação acadêmica. Cadernos EBAPE.BR, v. 9, n. 4, Opinião 3, p. 1174-1178, 2011. 
ASHKANASY, N. Publishing today is more difficult than ever. Journal of Organizational Behavior, v. 31, p. 1-3, 2010 .

BARBOSA, A. C. Q. Gestão de recursos humanos no Brasil: realidade atual e perspectivas. In: SILVA, J. R; BARBOSA, A. C. Q. (Orgs). Estado, empresas e sociedade: um mosaico luso-brasileiro. Lisboa: Colibri, 2009.

BARTUNEK, J. Academic-practitioner collaboration need not require joint or relevant research: Toward a relational scholarship of integration. Academy of Management Journal, v. 50, no 6, p. 1323-1333, 2007.

BARTUNEK, J; RYNES, S. The construction and contributions of "implications for practice": what's in them and what might they offer? Academy of Management Learning EEducation, v. 9, n. 1, p. 100-118, 2010.

BEECH, N; MACINTOSH, R; MACLEAN, D. Dialogues between academics and practitioners: the role of generative dialogic encounters. Organization Studies, v. 31, n. 9-10, p. $1341-1367,2010$

BERTERO, C. Editorial. RAE Eletrônica, v. 6, n. 1, 2007.

BERTERO, C; VASCONCELOS, F; BINDER, M. Estratégia empresarial: a produção científica brasileira entre 1991 e 2002. RAE-Revista de Administração de Empresas, São Paulo, v. 43, n. 4, p. 48-63, 2003.

BERTERO, C; CALDAS, M; WOOD JR, T. Produção científica em Administração de empresas: provocações, insinuações e contribuições para um debate local. Revista de Administração Contemporânea, v. 2, n. 1, p. 147-178, 1999.

Bertero, C; CALDAS, M; WOOD JR, T. Produção científica em Administração no Brasil: o estado da arte. São Paulo: Atlas, 2005.

BERTERO, C; KEINERT, T. A evolução da análise organizacional no Brasil (1961-93). RAE-Revista de Administração de Empresas, v. 34, n. 3, p. 81-90, 1994.

BEYER, J; CHANOVE, R; FOX, W. The review process and the fates of manuscripts submitted to AMJ. Academy of Management Journal, v. 38, nํ 5, p. 1219-1260, 1995.

CALDAS, M; TINOCO, T. Pesquisa em gestão de recursos humanos nos anos 1990: um estudo bibliométrico.
RAE-Revista de Administração de Empresas, v. 44, n. 3, p. 100-114, 2004.

CARVAlHO, C; VIEIRA, M. Algo está podre no reino da Dinamarca. Organizações e Sociedade, v. 10, n. 26, p. 185-187, 2003.

CLARK, T; FLOYD, S; WRIGHT, M. On the review process and journal development. Journal of Management Studies, v. 43, n. 3, p. 655-663, 2006

DAVEL, E; ALCADIPANI, R. Estudos críticos em Administração: reflexões e constatações sobre a produção brasileira. In: Encontro de Estudos Organizacionais, 2, 2002, Recife. Anais. Recife: Observatório da Realidade Organizacional - PROPAD/UFPE - ANPAD, 2002.

EDITORIAL TEAM. Information for contributors. Academy of Management Review, v. 50, n. 5, p. 979-981, 2007.

EISENHARDT, K. Better stories and better constructs: the case for rigor and comparative logic. Academy of Management Review, v. 16, n. 3, p. 620-627, 1991.

FREITAS, M. O pesquisador hoje: entre o artesanato intelectual e a produção em série. Cadernos EBAPE.BR, v. 9, n. 4, p. 1158-1163, 2011.

GEPHART, R. From the editors: qualitative research and the academy of management journal. Academy of Management Journal, v. 47, n. 4, p. 454-462, 2004.

GOLDEN-BIDDLE, K; LOCKE, K. Appealing work: an investigation of how ethnographic texts convince. Organization Science, v. 4, n. 4, p. 595-616, 1993.

GREY, C. Re-imagining relevance: a response to Starkey and Madan. British Journal of Management, v. 12, S27-S32. doi: 10.1111/1467-8551.12.s1.3, 2001.

ILGEN, D. Citations to management articles: cautions for the science about advice for the scientist. Academy of $M a-$ nagement Journal, v. 50, n. 3, p. 507-509, 2007.

JARZABKOWSKI, P; MOHRMAN, S; SCHERER, A. Organization studies as applied science: the generation and use of academic knowledge about organizations - introduction to the special issue. Organization Studies, v. 31, n. 9-10, p. 1189-1207, 2010. 
JARZABKOWSKI, P; WHITTINGTON, R. A strategy-as-practice approach to strategy research and education. Journal of Management Inquiry, v. 17, no 4, p. 282-286, 2008.

JUDGE, T; CABLE, D; COLBERT, A; RYNES, S. What causes a management article to be cited: article, author or journal? Academy of Management Journal, v. 50, n. 3, p. 491-506, 2007.

KNIGHTS, D; SCARBROUGH, H. In search of relevance: perspectives on the contribution of academic-practitioner networks. Organization Studies, v. 31, n. 9-10, p. 12871309, 2010.

LOCKE, K; GOLDEN-BIDDLE, K. Constructing opportunities for contribution: structuring intertextual coherence and "problematizing" in organizational studies. The Academy of Management Journal, v. 40, n. 5, p. 10231062, 1997.

MACHADO-DA-SILVA, C; GUARRIDO FILHO, E; ROSSONI, L; GRAEFF, J. Periódicos brasileiros de Administração: análise bibliométrica de impacto no triênio 2005-2007. RAC-Eletrônica, Curitiba, v. 2, n. 3, art. 1, p. 351-373, Set./ Dez. 2008

MASCARENHAS, A; ZAMBALDI, F; MORAES, E. Rigor, relevância e desafios da academia em Administração: tensões entre pesquisa e formação profissional. RAE-Revista de Administração de Empresas, v. 51, n. 3, p. 265-279, 2011.

MATTOS, P. Nós e os índices: a propósito da pressão institucional por publicação. RAE-Revista de Administração de Empresas, v. 48, n. 2, p. 144-149, 2008.

MATTOS, P. Pés de barro do texto "produtivista" na academia. RAE-Revista de Administração de Empresas, v. 52, n. 5, p. 566-573, 2012.

MEDEIROS, J; OLIVEIRA, J. Uma viagem à produção científica em qualidade de vida no trabalho (QVT) nos anos 2001-2005: estudo nos anais do Enanpad. Revista Eletrônica de Ciência Administrativa, v. 8, n. 1, p. 31-43, 2009.

NICOLAI, A; SEIDL, D. That's relevant! Different forms of practical relevance in management science. Organization Studies, v. 31, n. 9-10, p. 1257-1285, 2010.

PRATT, M. From the editors: for the lack of a boilerplate - tips on writing up (and reviewing) qualitative research.
The Academy of Management Journal, v. 52, n. 5, p. 856862, 2009.

RAE-REVISTA DE ADMINISTRAÇÃO DE EMPRESAS. Realizações em destaque. São Paulo: FGV-EAESP, 2012.

RODRIGUES, S. B. Comentando: Quem responde pelo desempenho limitado da produção científica em Administração no Brasil. Organizações e Sociedade, v. 11, n. 29, p. 193-196, 2004.

ROESCH, S. M. A. Quem responde pelo desempenho limitado da produção científica em Administração no Brasil. Organizações e Sociedade, v. 10, n. 28, p. 165$167,2003$.

ROUSSEAU, D; MANNING, J; DENYER, D. Evidence in management and organizational science: assembling the field's full weight of scientific knowledge through syntheses. AIM Working Paper Series 67, 2008. Disponível em: http://www.evidencebased-management.com/wp-content/ uploads/2010/01/ROUSSEAU-Evidence_2_15_08-11.pdf. Acesso em 23.03.2011.

RYNES, S. Let's create a tipping point: what academics and practitioners can do, alone and together. Academy of $\mathrm{Ma}$ nagement Journal, v. 50, n. 5, p. 1046-1054, 2007.

RYNES, S. From the editor: some reflections on contribution. Academy of Management Journal, v. 45, n. 2, p. 311-313, 2002.

RYNES, S; BARTUNEK, J; DAFT, R. Across the great divide: knowledge creation and transfer between practitioners and academics. Academy of Management Journal, v. 44, no 2 , p. 340-355, 2001.

SERRA, F; FIATES, G; FERREIRA, M. Publicar é difícil ou faltam competências? O desafio de pesquisar e publicar em revistas científicas na visão de editores e revisores internacionais. Revista de Administração Mackenzie, v. 9, n. 4, edição especial, p. 32-55, 2008.

SPINK, P; ALVES, M. O campo turbulento da produção acadêmica e a importância da rebeldia competente. Organizações E Sociedade, v. 18, n. 57, p. 337-343, 2011.

STARKEY, K; MADAN, P. Bridging the relevance gap: aligning stakeholders in the future of management research. British Journal of Management, v. 12, S3-S26, 2001. 
SUDDABY, R. From the editors: what grounded theory is not. Academy of Management Journal, v. 49, n. 4, p. 633642, 2006.

SUTTON, R; STAW, B. What theory is not. Administrative Science Quarterly, v. 40, n. 3, p. 371-384, 1995.

TONELLI, M; CALDAS, M; LACOMBE, B; TINOCO, T. O mapa da partilha: análise das áreas de comportamento organizacional e Gestão de Pessoas antes e depois da cisão da área de recursos humanos no Enanpad, 1991-2003. In: Encontro Anual da Associação Nacional de Programas de Pós-graduação em Administração, 21, 2004. Anais. Anpad, 2004.

TONELLI, M; CALDAS, M; LACOMBE, B; TINOCO, T. Produção acadêmica em recursos humanos no Brasil: 19912000. RAE-Revista de Administração de Empresas, v. 43, n. 1, p. 105-122, 2003.

VAN DE VEN, A. Engaged scholarship: a guide for organizational and social research. Oxford: Oxford University, 2007.

VASCONCELOS, F. Relevância e rigor na academia. RAE-Revista de Administração de Empresas, v. 49, n. 1, p. 5, 2009.

WEICK, K. Speaking to practice: the scholarship of integration. Journal of Management Inquiry, v. 5, $\mathrm{n}^{-}$1, p. 251-258, 1996.

WHETTEN, D. O que constitui uma contribuição teórica? RAE-Revista de Administração de Empresas, v. 43, n. 3, p. 69-73, 2003.

WOOD JR; TONELLI, M; COOKE, B. Colonização e neocolonização da gestão de recursos humanos no Brasil (19502010). RAE-Revista de Administração de Empresas, v. 51, n. 3, p. 232-243, 2011. 